\title{
The Association between Surgery Laterality in Orthopedists and the Choices of Prostheses in Total Knee and Hip Replacement
}

\author{
Jui-Yang Hsieh"1*, Ming-Shu Lan², Ying-Hsiu Chang', Jyh-Horng Wang4 \\ ${ }^{1}$ Department of Orthopedics, National Taiwan University Hospital and Jinshan Branch, Taiwan \\ ${ }^{2}$ Department of Orthopedics, Min-Sheng General Hospital, Taiwan \\ ${ }^{3}$ Department of Emergency, National Taiwan University Hospital Jinshan Branch, Taiwan \\ ${ }^{4}$ Department of Orthopedics, National Taiwan University Hospital, Taiwan \\ Email: *ocean_x5@yahoo.com.tw, otto_lan@yahoo.com.tw, Ashirly99@gmail.com,jhwang@ntuh.gov.tw
}

How to cite this paper: Hsieh, J.-Y., Lan, M.-S., Chang, Y.-H. and Wang, J.-H. (2018) The Association between Surgery Laterality in Orthopedists and the Choices of Prostheses in Total Knee and Hip Replacement. Open Journal of Orthopedics, 8, 257-266. https://doi.org/10.4236/ojo.2018.87029

Received: May 15, 2018

Accepted: July 1, 2018

Published: July 4, 2018

Copyright $\odot 2018$ by authors and Scientific Research Publishing Inc. This work is licensed under the Creative Commons Attribution International License (CC BY 4.0).

http://creativecommons.org/licenses/by/4.0/

\begin{abstract}
Background: Most orthopedists are right-handed. However, osteoarthritis occurs with equal frequency over both sides. Does perfect and effective arthroplasty surgery require ambidextrous motor skills? Objectives: In this study, we aimed to investigate the clinical features of arthroplasty for hip and knee joints (THR and TKR) on different sides affected by orthopedist laterality. Patients and Methods: All right-handed orthopedists performed 64 right and 52 left primary THR among 100 patients, and 115 right and 118 left primary TKR among 192 patients. Clinical and surgical features were retrospectively reviewed and analyzed. The body mass index (BMI), inner diameter of acetabular cup liners (ACD), diameter of the femoral head (FHD), diameter of the femoral stem (FSD), and the femoral neck length (FNL) were included in THR-receiving patients. The BMI, femoral component (FC) size, tibial plate (TP) size, and thickness of articular surface inserts (ASI) were included in TKR-receiving patients. Results: No significant differences were observed in ACD, FHD and FSD between patients receiving right and left THR groups ( $p$ $=0.16,0.11$, and 0.05 , respectively). Similarly, there were no significant differences in FC, TP, and ASI between patients receiving right and left TKR groups ( $p=0.06,0.80$, and 0.46 , respectively). However, FNL in left THR group was significantly longer than that in right THR group $(p=0.01)$. Conclusion: This study showed that dexterity or proprioception in handedness had no affect on bone resection and thicknesses of the polyethylene in TKR and acetabular reaming and femoral canal rasping in THR. However, it did lead to differences in femoral neck resection. Right-handed orthopedists significantly tend to perform excessive femoral neck cutting or prefer the larger
\end{abstract}


size of FNL when performing left side THR, while no differences occur when performing TKR.

\section{Keywords}

Total Hip Replacement, Total Knee Replacement, Handedness, Laterality

\section{Introduction}

Most people and surgeons are right-handed. Handedness has been reported to play a major role in surgical outcomes [1] [2] [3] [4]. Medical students discordant with their hand dominance may have inferior acquisition of basic surgical skills [5]. Both hands may result in similar efficiency to perform endotracheal intubation by an experienced anesthesiologist using advanced instruments [6]. However, handedness appears to have an influence over the performance in high surgical skills due to absence of comfort and ergonomy, such as laparoscopic cholecystectomy, dental therapy [7] [8]. Poor insertion of the sliding hip screw and pedicle screws is more commonly found on the left side when the surgeon is right-handed [9] [10]. Osteoarthritis occurs equally on both sides, but laterality of surgeons might influence the clinical features of orthopedic surgery. In addition, handedness has been reported to play a major role in outcomes of total knee replacement (TKR) by right-handed orthopedic surgeons with a significantly better function and pain scores for right knees than for left knees one year after surgery [11].

Arthroplasty in orthopedic surgery is a highly technically demanding procedure, especially due to the symmetrical disposition of the anatomy and the potential complications of discrepancies in the leg length after surgery. However, whether objective and quantitative laterality-based differences occur with right-handed surgeons is unclear.

Is there a statistically significant difference between the two sides for the orthopedic surgery with better performance when the surgical laterality was located in the dominant hand? Therefore, the aim of this study was to evaluate the outcomes between right and left total hip/knee replacements (THR/TKR) by right-handed orthopedic surgeons.

\section{Methods}

Due to funding, manpower and administration, we retrospectively reviewed the medical records at Min-Sheng General Hospital over a 7-year period from January 2004 to June 2011. So there were 212 patients underwent primary THR for osteoarthritis of the hip or femoral head necrosis, and 312 patients underwent primary TKR for osteoarthritis of the knee. The Institutional Review Board of Min-Sheng General Hospital approved this retrospectively investigational study (No. 2012005 and No. 2012006) on Aug. 15 ${ }^{\text {th }}, 2012$ and Aug. $27^{\text {th }}, 2012$. 
The exclusion criteria in the THR groups were revision, incompletely recorded data, and a primary diagnosis of bone tumor or infection. After screening, 100 patients received 116 THR (Versys Hip System, Zimmer Inc., Warsaw, IN, USA) were included in the current study. During the study period, all THR procedures were performed by 18 right-handed orthopedists. The exclusion criteria in TKR groups similarly comprised of revision, incompletely recorded data, and a primary diagnosis of bone tumor or infection. A total of 192 patients who met the criteria were identified, receiving 233 TKR using the NexGen ${ }^{\circledR}$ Legacy Posterior-Stabilized Flex system (Zimmer Inc., Warsaw, IN, USA). During the study period, all TKR procedures were performed by 16 right-handed orthopedists.

The inner diameter of acetabular cup liners (ACD), diameter of the femoral head (FHD), diameter of the femoral stem (FSD), and the femoral neck length (FNL) of the implanted THR were collected from medical records in THR groups. The size of the femoral component (FC), size of the tibial plate (TP) and thickness of articular surface inserts (ASI) of the implanted TKR were collected from medical records in TKR groups. The body mass index (BMI) of the patients was also calculated using body weight and height. The correlation between BMI, and ACD, FHD, FSD, FNL in THR group and between BMI and FC, TP, ASI in TKR group were analyzed with regards to laterality.

The data were evaluated using the chi-square test for gender and the test for age and size of the prostheses. The level of statistical significance was set at $p<$ 0.05 .

\section{Results}

The characteristics of the patients are presented in Table 1 and Table 2. Twenty women and 44 men received right THR with an average age of 54.1 years and a BMI of $24.4 \mathrm{~kg} / \mathrm{m}^{2}$. Twelve women and 40 men received left THR with an average age of 55.1 years and a BMI of $24.7 \mathrm{~kg} / \mathrm{m}^{2}$. There were no significant differences in gender, age and BMI between the two groups $(p=0.33,0.37$, and 0.35 , respectively).

Eighty-four women and 31 men received right TKR with an average age of 67.2 years and a BMI of $28.5 \mathrm{~kg} / \mathrm{m}^{2}$. Eighty-one women and 37 men received left TKR with an average age of 69.7 years and a BMI of $28.0 \mathrm{~kg} / \mathrm{m}^{2}$. There were no significant differences in gender, age and BMI between the two groups $(p=0.47$, 0.07 , and 0.44 , respectively).

Table 1. The patient characteristics in the THR group.

\begin{tabular}{cccccccc}
\hline & $\begin{array}{c}\text { Gender } \\
(\text { Female/Male })\end{array}$ & $\begin{array}{c}\text { Age (years } \\
\text { old })\end{array}$ & $\begin{array}{c}\text { BMI } \\
\left(\mathrm{kg} / \mathrm{m}^{2}\right)\end{array}$ & ACD $(\mathrm{mm})$ & FHD $(\mathrm{mm})$ & FSD $(\mathrm{mm})$ & FNL $(\mathrm{mm})$ \\
\hline Right & $20 / 44$ & $54.1 \pm 15.8$ & $24.4 \pm 3.9$ & $53.4 \pm 3.1$ & $29.8 \pm 5.8$ & $12.4 \pm 1.6$ & $0.08 \pm 2.49$ \\
Left & $12 / 40$ & $55.1 \pm 15.8$ & $24.7 \pm 3.5$ & $54.0 \pm 2.9$ & $28.3 \pm 2.5$ & $12.8 \pm 1.5$ & $1.21 \pm 2.90$ \\
$P$ Value & $0.33^{*}$ & $0.37^{\dagger}$ & $0.35^{\dagger}$ & $0.16^{\dagger}$ & $0.11^{\dagger}$ & $0.05^{\dagger}$ & $0.01^{\dagger}$ \\
\hline
\end{tabular}

${ }^{*}$ The chi-square tests. ${ }^{\dagger}$ The $t$ test. 
Table 2. The patient characteristics in the TKR group.

\begin{tabular}{ccccccc}
\hline & $\begin{array}{c}\text { Gender } \\
(\text { Female/Male })\end{array}$ & Age (years old) & BMI $\left(\mathrm{kg} / \mathrm{m}^{2}\right)$ & FC $\left(\mathrm{mm}^{2}\right)$ & TP $\left(\mathrm{mm}^{2}\right)$ & ASI $(\mathrm{mm})$ \\
\hline Right & $84 / 31$ & $67.2 \pm 9.5$ & $28.5 \pm 4.8$ & $3760.8 \pm 384.1$ & $2964.2 \pm 318.5$ & $11.0 \pm 1.6$ \\
Left & $81 / 37$ & $69.7 \pm 8.5$ & $28.0 \pm 4.5$ & $3890.2 \pm 384.1$ & $2974.9 \pm 316.4$ & $10.8 \pm 1.5$ \\
$P$ Value & $0.47^{*}$ & $0.07^{\dagger}$ & $0.44^{\dagger}$ & $0.06^{\dagger}$ & $0.80^{\dagger}$ & $0.46^{\dagger}$ \\
\hline
\end{tabular}

${ }^{*}$ The chi-square tests. ${ }^{\dagger}$ The $t$ test.

In regard to the prostheses, no significant differences were observed in ACD, FHD and FSD between patients receiving right and left THR groups ( $p=0.16$, 0.11 , and 0.05 , respectively). Similarly, there were no significant differences in FC, TP, and ASI between patients receiving right and left TKR groups ( $p=0.06$, 0.80 , and 0.46 , respectively). However, the average FNL was $0.08 \mathrm{~mm}$ in right THR group and $1.21 \mathrm{~mm}$ in left THR group. The FNL was significantly longer in the left THR group than in right THR group $(p=0.01)$, however the correlation coefficient between BMI and FNL was weak $(\mathrm{r}=0.03)$.

The average ACD was $53.4 \mathrm{~mm}$ in right THR group and $54.0 \mathrm{~mm}$ in left THR group. The average FHD was $29.8 \mathrm{~mm}$ in right THR group and $28.3 \mathrm{~mm}$ in left THR group. The average FSD was $12.4 \mathrm{~mm}$ in right THR group and $12.8 \mathrm{~mm}$ in left THR group. The correlation coefficients between BMI and ACD, FHD and FSD were all weak ( $\mathrm{r}=-0.14,-0.12$, and -0.12 , respectively).

The cross-sectional area of the femoral component ranged from size $\mathrm{C}$ to $\mathrm{F}$ is approximate calculation by its length and width, $3270 \mathrm{~mm}^{2}$ in size C, 3756.8 $\mathrm{mm}^{2}$ in size D, $4250 \mathrm{~mm}^{2}$ in size E, and $4788 \mathrm{~mm}^{2}$ in size F. The cross-sectional area of the tibial plate ranged from size 2 to 6 , with $2542 \mathrm{~mm}^{2}$ in size 2, 2772 $\mathrm{mm}^{2}$ in size 3, $3036 \mathrm{~mm}^{2}$ in size 4, $3404 \mathrm{~mm}^{2}$ in size 5 , and $3700 \mathrm{~mm}^{2}$ in size 6 .

The average cross-sectional area of FC was $3760.8 \mathrm{~mm}^{2}$ in right TKR group and $3890.2 \mathrm{~mm}^{2}$ in left TKR group. The average cross-sectional area of TP was $2964.2 \mathrm{~mm}^{2}$ in right TKR group and $2974.9 \mathrm{~mm}^{2}$ in left TKR group. The average thickness of ASI was $11.0 \mathrm{~mm}$ in right TKR group and $10.8 \mathrm{~mm}$ in left TKR group. The correlation coefficients between BMI and FC, BMI and TP, BMI and ASI were all weak $(r=0.05,-0.02$, and 0.02 , respectively).

\section{Discussion}

The body size of our patients generally was not particularly obese compared to other studies [12] [13]. In addition to, there was no significant difference in BMI between the right and left TKR groups. Several studies have shown that obesity is a risk factor for more intraoperative difficulties and more surgical complications [14] [15], whereas others have shown no differences in the overall complication rates or implant survival [16] [17]. Whether a high BMI results in an increased rate of post-operative prosthetic complications following THR is controversial [18] [19]. The correlation between BMI and THR or TKR prosthesis size was low in this study. 
A proper femoral neck cut to restore anatomic parameters during THR surgery may be influenced by many factors. It is important that the sure of the cutting angle was inclined by $45^{\circ}$ to the centerline of the femur in the meanwhile [20]. A standard FNL size of $0 \mathrm{~mm}$ in Zimmer THR prostheses for the appropriate choice of femoral neck resection levels is recommended to be $15 \mathrm{~mm}$ above the lesser trochanter. All of the orthopedic surgeons have received thorough orthopedic training and were supposed to give full attention to achieve the optimal size of the femoral neck cut and femoral stem positioning.

The femoral cut depends on the surgeon's preference, and can involve soft tissue balance, and initial acetabular condition or leg length discrepancy. The average FNL in the left THR group $(1.21 \mathrm{~mm})$ was significantly longer than that in the right THR group $(0.08 \mathrm{~mm})$ except for differences between the stated FNL and the actual length of the implants supplied by the manufacturers. The correlation coefficients between BMI and FNL, ACD, FHD and FSD were all weak, so the patient's body size may not affect the decision making with regards to femoral neck cutting level or other THR procedures. In contrast, laterality-based differences with the non-dominant side should remind right-handed orthopedic surgeons not to perform excessive femoral cutting during left THR.

There are several major bone cuts in a TKR under the use of customized jigs to enable a more stable fixation of the cutting blocks and minimize cutting errors [21] [22]. The accuracy of proximal tibia resections can be affected by anatomic variations [23]. There were no significant differences in BMI between the two TKR groups, so there may be a less mass effect in the extremities for proximal tibia cutting. There seemed to be minimal differences in tibial resection and femoral cutting between the two groups as there were no significant differences in the cross-sectional areas of FC and TP ( $p=0.06$ and 0.80 , respectively). Therefore, the right-handed orthopedic surgeons performed precisely the bony procedures of tibial resection and femoral cutting between right and left TKR groups in our study.

Except for adequate bone resection, choosing the optimal minimal insert thickness of the polyethylene for soft-tissue balance to achieve compromise during TKR procedures is every orthopedic surgeon's mission [24] [25]. The average thickness of ASI was not significantly different between the two groups ( $p$ $=0.46)$, and the correlation coefficient between BMI and ASI was also weak $(\mathrm{r}=$ -0.02). Therefore, the right-handed orthopedic surgeons successfully performed TKR procedures to achieve bone resection, soft-tissue balance and thicknesses of the polyethylene between the two groups.

All THR and TKR procedures in this study were performed by right-handed surgeons, which may have biased the surgical outcomes. When the right-handed orthopedic surgeon stands behind the patient to perform right THR, the deviation angle between the axis of the surgeon's right forearm and the line of patient's right femoral neck osteotomy projected onto the horizontal plane is usually parallel or mild deviated (Figure 1). However, in left THR procedures, the deviation angle is approximate perpendicular (Figure 2). A clearer and 


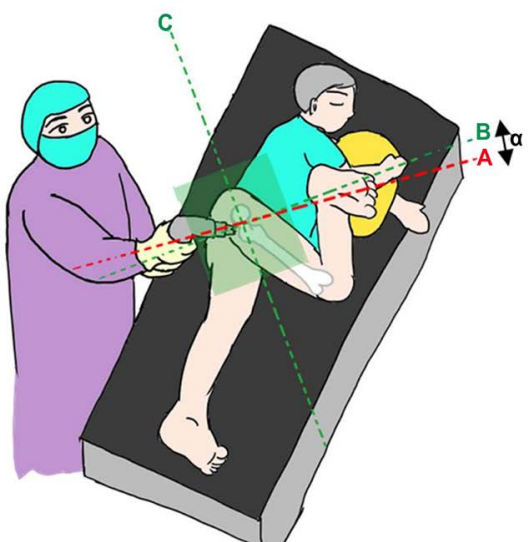

(a)

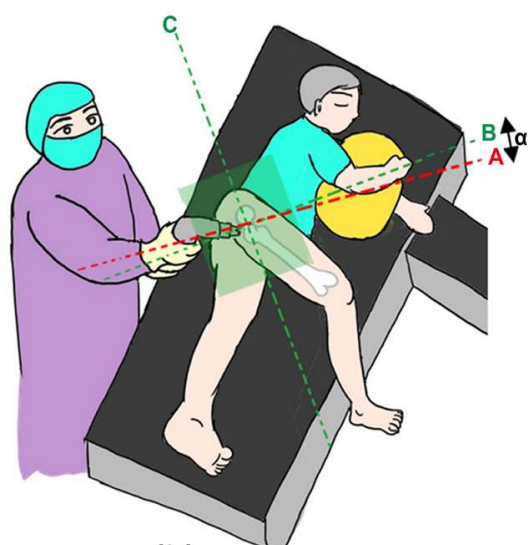

(b)

Figure 1. The schematic diagram of the deviation angle $(\alpha)$ during right THR procedures. The deviation angle is between the projected axis of the surgeon's right forearm (the red dotted line A) and the projected line of femoral neck osteotomy (the green dotted line B) on the horizontal plane (the green rectangle) under the posterolateral approach (a) or the anterolateral approach (b). The line A is obviously closer to the line B and far from away the line $\mathrm{C}$ (the axis of femoral head center to neck projected onto the green rectangle).

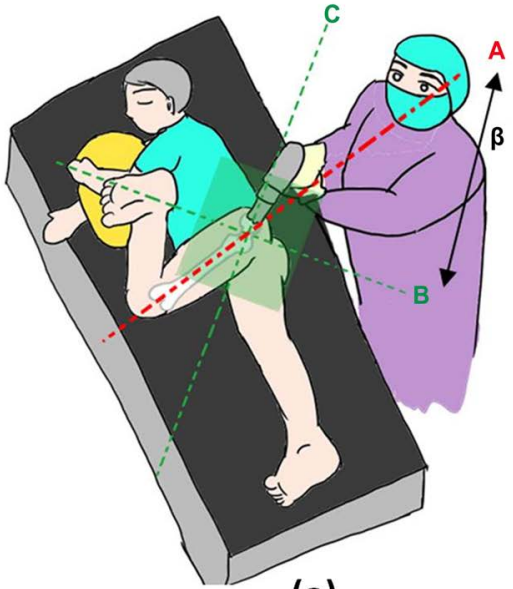

(a)

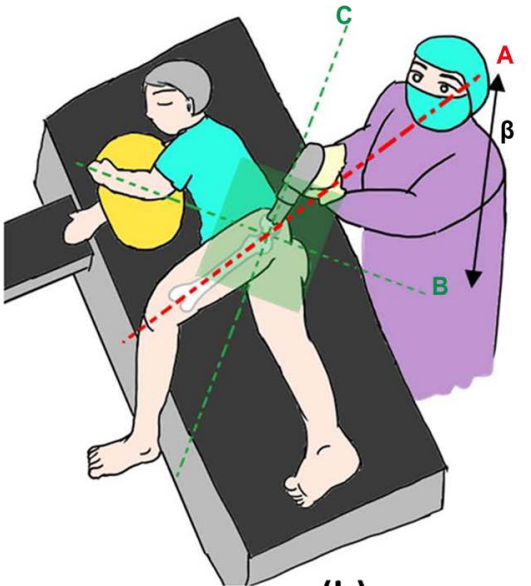

(b)

Figure 2. The schematic diagram of the deviation angle $(\beta)$ during left THR procedures. The deviation angle is between the axis of the surgeon's right forearm (the red dotted line A) and the line of femoral neck osteotomy (the green dotted line B) projected onto the horizontal plane (the green rectangle) under the posterolateral approach (a) or the anterolateral approach (b). The line A is between the line B and the line $\mathrm{C}$ (the axis of femoral head center to neck projected onto the green rectangle).

better view of being going to be preserved femoral shaft and basal femoral neck during right-handed surgeon performing right THR lead to more cautious femoral neck cutting or more precise size of FNL chosen compared to left THR. Left-sided THR is technically more difficult for a right-handed surgeon due to anatomical reasons, which may lead to right-handed orthopedic surgeons performing excessive femoral cutting or preferring the larger size of FNL.

The axis of the surgeon's right forearm is somewhat more deviated to the patient's tibial axial projected onto the plane of tibial osteotomy surface in the left 
TKR group compared to that in the right TKR group (Figure 3 and Figure 4). The projected errors in the mechanical axes of the femur and tibia due to errors during TKR procedures are unlikely to bear clinical significance [26]. In addition, good exposure and a clear view of the distal femur and proximal tibia in both sides of TKR result in fewer resection errors. Left-sided TKR is technically no more difficult for a right-handed surgeon even with anatomic barriers, as evidenced by there being no significant differences in the size of TKR components in FC, TP and ASI between the two groups.

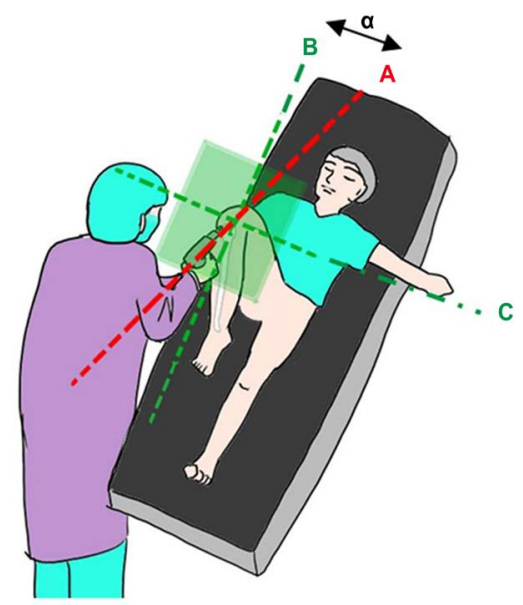

Figure 3. The schematic diagram of the deviation angle $(\alpha)$ during right TKR procedures. The deviation angle is between the projected axis of the surgeon's right forearm (the red dotted line A) and the projected axis of the patient's right tibia (the green dotted line $\mathrm{B}$ ) on the tibial osteotomy plane (the green rectangle). The line $\mathrm{A}$ is between the line $\mathrm{B}$ and the line $\mathrm{C}$ (the perpendicular line to the line $\mathrm{B}$ on the green rectangle) and is often close to the line $\mathrm{B}$.

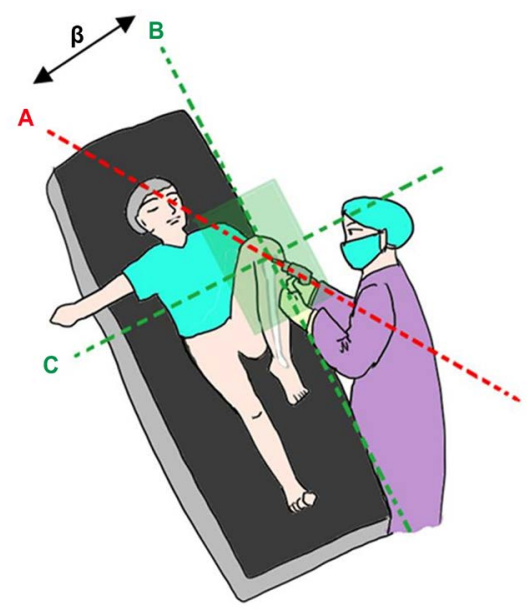

Figure 4. The schematic diagram of the deviation angle $(\beta)$ during left TKR procedures. The deviation angle is between the projected axis of the surgeon's right forearm (the red dotted line A) and the projected axis of the patient's left tibia (the green dotted line B) on the tibial osteotomy plane (the green rectangle). The line $\mathrm{A}$ is between the line $\mathrm{B}$ and the line $\mathrm{C}$ (the perpendicular line to the line $\mathrm{B}$ on the green rectangle) and is not particular close to the line B or line $\mathrm{C}$. 
There are several limitations to this study. First, it is retrospective in design. Second, our findings do not explain the detailed mechanism accounting for a longer FNL or excess femoral neck resection on the left side. Third, this study does not compare patients who received anterolateral or posterolateral approaches for THR procedures. Finally, this study did not gather data of patients with valgus or varus knees.

\section{Conclusion}

This study showed that dexterity or proprioception in handedness had no affect on bone resection and thicknesses of the polyethylene in TKR procedures and acetabular reaming and femoral canal rasping in THR procedures. However, it did lead to differences in femoral neck resection. Right-handed orthopedists are significantly more likely to perform excessive femoral neck cutting or prefer the larger size of FNL when performing left side THR surgery, while no differences occur when performing TKR surgery. The surgeon should be aware of the potential ambiguity and practice THR more meticulously to make perfect.

\section{Conflict of Interest}

The authors state that there is no conflict of interest. The patient was informed and gave contentment for this article.

\section{Author's Contribution}

All the authors contributed to the writing of this manuscript, and had read and approved the final version.

\section{References}

[1] Pedowitz, R., Nicandri, G. and Tuchschmid, S. (2016) Asymmetry in Dominant/Non-Dominant Hand Performance Differentiates Novices from Experts on an Arthroscopy Virtual Reality Serious Game. Studies in Health Technology and Informatics, 220, 289-294.

[2] Flatt, A.E. (2008) Is Being Left-Handed a Handicap? The Short and Useless Answer is "Yes and No." Baylor University Medical Center Proceedings, 21, 304-307.

[3] Tchantchaleishvili, V. and Myers, P.O. (2010) Left-Handedness-A Handicap for Training in Surgery? Journal of Surgical Education 67, 233-236. https://doi.org/10.1016/j.jsurg.2010.06.001

[4] Hanna, G.B., Drew, T., Clinch, P., Shimi, S., Dunkley, P., Hau, C. and Cuschieri, A. (1997) Psychomotor Skills for Endoscopic Manipulations: Differing Abilities between Right and Left-Handed Individuals. Annals of Surgery, 225, 333-338. https://doi.org/10.1097/00000658-199703000-00014

[5] Lee, J.Y., Mucksavage, P. and McDougall, E.M. (2013) Surgical Skills Acquisition among Left-Handed Trainees-True Inferiority or unfair Assessment: A Preliminary Study. Journal of Surgical Education, 70, 237-242. https://doi.org/10.1016/j.jsurg.2012.09.007

[6] Kuo, Y.W., Yen, M.K., Cheng, K.I., Tang, C.S., Chau, S.W., Hou, M.F., Wang, J.J. and Lin, S.F. (2007) Lightwand-Guided Endotracheal Intubation Performed by the 
Nondominant Hand Is Feasible. The Kaohsiung Journal of Medical Sciences, 23, 504-510. https://doi.org/10.1016/S1607-551X(08)70008-1

[7] Oms, L.M. and Badia, J.M. (2003) Laparoscopic Cholecystectomy In Situs Inversus Totalis: The Importance of Being Left-Handed. Surgical Endoscopy, 17, 1859-1861. https://doi.org/10.1007/s00464-003-9051-7

[8] Canakci, V., Tan, U., Orbak, R. and Tezel, A. (2002) Right- and Left-Handed Dentists in Periodontal Therapy. International Journal of Neuroscience, 112, 1-14.

[9] Yaman, O. and Acaroğlu, E. (2014) Role of Surgeon Handedness in Transpedicular Screw Insertion. Acta Orthopaedica et Traumatologica Turcica, 48, 479-482. https://doi.org/10.3944/AOTT.2014.13.0046

[10] Moloney, D., Bishay, M., Ivory, J. and Pozo, J. (1994) Failure of the Sliding Hip Screw in the Treatment of Femoral Neck Fractures: "Left-Handed Surgeons for Left-Sided Hips”. Injury, 25, B9-B13. https://doi.org/10.1016/0020-1383(94)90194-5

[11] Mehta, S. and Lotke, P.A. (2007) Impact of Surgeon Handedness and Laterality on Outcomes of Total Knee Arthroplasties: Should Right-Handed Surgeons Do only Right TKAs? American Journal of Orthopedics, 36, 530-533.

[12] Spicer, D.D., Pomeroy, D.L., Badenhausen, W.E., Schaper, L.A., Curry, J.I., Suthers, K.E. and Smith, M.W. (2001) Body Mass Index as a Predictor of Outcome in Total Knee Replacement. International Orthopaedics, 25, 246-249. https://doi.org/10.1007/s002640100255

[13] Si, H.B., Zeng, Y., Shen, B., Yang, J., Zhou, Z.K., Kang, P.D. and Pei, F.X. (2015) The Influence of Body Mass Index on the Outcomes of Primary Total Knee Arthroplasty. Knee Surgery, Sports Traumatology, Arthroscopy, 23, 1824-1832.

[14] Liabaud, B., Patrick, D.A. and Geller, J.A. (2013) Higher Body Mass Index Leads to Longer Operative Time in Total Knee Arthroplasty. The Journal of Arthroplasty, 28, 563-565. https://doi.org/10.1016/j.arth.2012.07.037

[15] Liljensøe, A., Lauersen, J.O., Søballe, K. and Mechlenburg, I. (2013) Overweight Preoperatively Impairs Clinical Outcome after Knee Arthroplasty: A Cohort Study of 197 Patients 3-5 Years after Surgery. Acta Orthopaedica, 84, 392-397. https://doi.org/10.3109/17453674.2013.799419

[16] Jackson, M.P., Sexton, S.A., Walter, W.L., Walter, W.K. and Zicat, B.A. (2009) The Impact of Obesity on the Mid-Term Outcome of Cementless Total Knee Replacement. The Journal of Bone and Joint Surgery. British Volume, 91, 1044-1048.

[17] Collins, R.A., Walmsley, P.J., Amin, A.K., Brenkel, I.J. and Clayton, R.A. (2012) Does Obesity Influence Clinical Outcome at Nine Years Following Total Knee Replacement? The Journal of Bone and Joint Surgery. British Volume, 94, 1351-1355. https://doi.org/10.1302/0301-620X.94B10.28894

[18] Davis, A.M., Wood, A.M., Keenan, A.C., Brenkel, I.J. and Ballantyne, J.A. (2011) Does Body Mass Index Affect Clinical Outcome Post-Operatively and at Five Years after Primary Unilateral Total Hip Replacement Performed for Osteoarthritis? A Multivariate Analysis of Prospective Data. The Journal of Bone and Joint Surgery. British Volume, 93, 1178-1182. https://doi.org/10.1302/0301-620X.93B9.26873

[19] McLaughlin, J.R. and Lee, K.R. (2006) The Outcome of Total Hip Replacement in Obese and Non-Obese Patients at 10- to 18-Year. The Journal of Bone and Joint Surgery. British Volume, 88, 1286-1292. https://doi.org/10.1302/0301-620X.88B10.17660

[20] Mihalko, W.M., Saleh, K.J., Heller, M.O., Mollard, B., König, C. and Kammerzell, S. (2009) Femoral Neck Cut Level Affects Positioning of Modular Short-Stem Implant. 
Orthopedics, 32, 18-21. https://doi.org/10.3928/01477447-20090915-53

[21] Brooks, P. (2009) Seven Cuts to the Perfect Total Knee. Orthopedics, 32, 680-682. https://doi.org/10.3928/01477447-20090728-27

[22] Nogler, M., Hozack, W., Collopy, D., Mayr, E., Deirmengian, G. and Sekyra, K. (2012) Alignment for Total Knee Replacement: A Comparison of Kinematic Axis versus Mechanical Axis Techniques. A Cadaver Study. International Orthopaedics, 36, 2249-2253. https://doi.org/10.1007/s00264-012-1642-2

[23] Chiu, K.Y., Yau, W.P., Ng, T.P. and Tang, W.M. (2008) The Accuracy of Extramedullary Guides for Tibial Component Placement in Total Knee Arthroplasty. International Orthopaedics, 32, 467-471. https://doi.org/10.1007/s00264-007-0354-5

[24] Edwards, S.A., Pandit, H.G., Ramos, J.L. and Grover, M.L. (2002) Analysis of Polyethylene Thickness of Tibial Components in Total Knee Replacement. The Journal of Bone and Joint Surgery, 84, 369-371. https://doi.org/10.2106/00004623-200203000-00006

[25] Pijls, B.G., Van der Linden-Van der Zwaag, H.M. and Nelissen, R.G. (2012) Polyethylene Thickness Is a Risk Factor for Wear Necessitating Insert Exchange. International Orthopaedics, 36, 1175-1180. https://doi.org/10.1007/s00264-011-1412-6

[26] Yau, W.P., Leung, A., Liu, K.G., Yan, C.H., Wong, L.L. and Chiu, K.Y. (2007) Interobserver and Intra-Observer Errors in Obtaining Visually Selected Anatomical Landmarks during Registration Process in Non-Image-Based Navigation-Assisted Total Knee Arthroplasty. The Journal of Arthroplasty, 22, 1150-1161.

https://doi.org/10.1016/j.arth.2006.10.010 